\title{
Spectrum Allocation in Cognitive Radio - Simplified Swarm Optimization Based Method
}

\author{
Rajalakshmi, P.Sumathy
}

\begin{abstract}
Communication through wireless mode is accelerated its expansion in broad manner that make a way to communicate with different type of computing devices to interact each other. As the number of users continues to increase, there is a constant demand for the usability of radio spectrum, which is a limited resource.Therefore a maximum utilization of spectrum is necessary at any moment. Moreover it is desired to share the capacity of the bandwidth between the user's application on the basis of different channel utilization without compromising efficiency and fairness. Because cognitive system accommodate a dynamic spectrum allocation environment and it becomes an essential to compare performance in terms of Throughput, Latency, End-To-End Delay, Average Power Consumption, Average Adaptation Time and Average Total Utility were provided to illustrate the improved behaviour of the proposed system. A quality conscious spectrum assessment work is proposed, where spectrum bands are examined based on the requirements of application as well as the complex existence of the spectrum bands. The author used Simplified Swam Optimization (SSO) in this paper to communicate spectrum allocation and the performance of the proposed method is compared with the existing methods; Genetic Algorithm (GA) and Particle Swam Optimization (PSO). It has been found that SSO gives an optimal solution than GA and PSO.
\end{abstract}

Key Words: Cognitive Radio, Spectrum Sharing, Genetic Algorithm, Particle Swam Optimization and Simplified Swam Optimization.

\section{INTRODUCTION}

Now-a-days communication network becomes the predominant due to multimedia applications where it requires a higher bandwidth and faster data rates [11]. The multimedia applications bring in different quality of services through third generation and fourth generation in order to achieve faster data rate in their allowed bandwidth requirements. The multimedia service and their information accessing convenience boost the development of the Wireless Multimedia. The patterns and efficiency of various devices have been heavily influenced by using higher bandwidth which is considered as a serious problem in the coming years. So, the next generation communication network has been designed to deal more efficiently with the explosive growth of data traffic and the emerging new technology scenarios in the future [5][10].

Revised Manuscript Received on February 05, 2020.

* Correspondence Author

Rajalakshmi*, Scholar, Department of Computer Science, Bharathidasan University, Trichy, Tamil Nadu, India - 620023, laks2020cs@gmail.com

Dr.P.Sumathy, Assisatnt Professor, Department of Computer Science,

Bharathidasan University, Trichy, Tamil Nadu, India - 620023, sumathy.p@bdu.ac.in

(C) The Authors. Published by Blue Eyes Intelligence Engineering and Sciences Publication (BEIESP). This is an open access article under the CC BY-NC-ND license (http://creativecommons.org/licenses/by-nc-nd/4.0/)
Thus, the expansion of communication network is required to accommodate the growth of the network communication which will be concentrated based on the capacity of the network. Next generation wireless networks have simple distributed controls and portable infrastructures which incorporate the technical issues related to the capability of the communication network where the feasibility and the cost of these techniques will be determined based on the growing demand [13]. While some society have suggested to maximize the bandwidth capacity in terms of Transmission Efficiency Per Megahertz (MHz) of spectrum and to improve the regional utilization of the spectrum which will be sufficient to meet the demand in the future. The basic techniques for expanding these wireless communication network capacity is necessary to include the expansion of the overall available radio spectrum [11]. The allocation of wireless frequency spectrum is considered to be the most challenging issue based on the demand and the limitations. The Graphical User Interface (GUI) is the virtual program fr amework that allows the user to interact with other gadgets based on different computational techniques. GUI has been used in different ways from standard computer to mobile devices including very small devices like watches[1] [7]. When different types of GUI arise, then its become an essential component to be checked to ensure the application achieves the user's desired performance. The cognitive system provides a dynamic spectrum allocation environment which brings necessary parameters such as Power Consumption, Throughput, Latency, End-to-End Delay, Average Power Consumption, Average Adaptation Timeand Average Total Utility. Unlike non-functional testing, GUI function testing ensures an appropriate interaction between the user and the interface of the application environment without dealing with the internal coding. Techniques for GUI functions are introduced using Genetic Algorithm (GA), Particle Swarm Optimization (PSO) and Simplified Swarm Optimization(SSO) are compared with each other.The effectiveness of the algorithm is demonstrated in the form of a graph by evaluating the parameters.

\section{BACKGROUND WORK}

Subhashree Mishra et al, proposed an algorithm for Cognitive Radio System where most of the communication taking place through a Multi-Carrier System. Here the cognitive system provides an environment of dynamic spectrum allocation [12]. 
Koroupi et al, suggested an innovative technique for utilizing Ant Colony System (ACS) and Fuzzy Logic (FL) which predominates communication networks suffering from lack of bandwidth and insufficiency of the spectrum [4]. Zhao et al suggested an interference synchronization mechanism to control spectrum sharing for Cognitive Radio (CR) networks, which is considered to be a powerful inference control strategy [8]. Yao Wang et al proposes a Cost and Connection Degree (CCB) algorithm that creates a new useful method to build a prototype to represent the overall utility of the process [14]. P.Vijayakumar and S.Malarvihi propose a Multiple-Input Multi-Output (MIMO) cognitive radio system with GA based power control, antenna selection and connection adoption are proposed to share the spectrum with minimal assurance [9]. FarokhKoroupi et al suggested a promising spectrum sharing clarification which avoids high spectrum costs and improving the utilization of spectrum resources, particularly Wider-Coverage, Massive-Capacity, Massive-Connectivity and Low-Latency [3]. The need for spectrum capacity is constantly increasing with the advancement of wireless communication technologies and the lack of wireless spectrum resources where Cognitive Radio has described as essential component which formulates an alternate way to avail the entire spectrum resources need on demand efficiently. Cognitive Radio networks consist of highly spaced base stations, numerous clients and sensor nodes. Spectrum allocation of secondary nodes is influenced by a variety of factors, which includes bit error rate, availability of free carriers, transmitting capacity and consumer need, etc., The flexibility exposure to the spectrum is its essential concept and it can use the unused spectrum efficiently without compromising user rights as many clients will share a part of the spectrum.

\section{SIMPLIFIED SWARM OPTIMIZATION (SSO)}

An integrated spectrum distribution system for Cognitive Radio Networks (CRN) is formed in which spectrum bands are defined with the existences of spectrum band by considering the application requirements [6]. Moreover, subcarrier allocation has been designed to meet the different performance based on Simplified Swarm Optimization Algorithm for Cognitive Radio users in CRNs. Selection of channels is essential which enables the CR to select the best channel among the sensed channels collected where the CR must able to adjust dynamically with the physical parameters such as bandwidth, strength and activity. Initially, the proposed scheme detects the reliability of the primary users which operates with multiple Primary Users (PU) networks. In the typical PSO, each particle has its own fitness value, which is determined by a fitness function at its current position in order to be optimize. PSO starts with the initial population of random particles with random positions and velocities which are updated from iteration-to-iteration within problem space. The particles travel the iteration in a multidimensional search space with their velocities constantly being updated by the perception of the particle itself. In Simplified Swarm Optimization (SSO) exchanges the local search where by initializing the a potential spectrum sharing system. It is fundamentally

amount of swarm population size, the cumulative generation number and three preset parameters are initially been calculated. The position value of the particle in each dimension will be maintained or updated by its pbest value or the gbest value in each generation or replaced by a new random value in accordance with this procedure[2]. The following equation is used to calculate the best fitness value.

$$
x_{i d}^{t}=\left\{\begin{array}{c}
x_{i d}^{t-1}, \text { if } \operatorname{rand}() \in\left[0, c_{w}\right], \\
p_{i d}^{t-1}, \text { if } \operatorname{rand}() \in\left[c_{w}, c_{p}\right), \\
g_{i d}^{t-1}, \text { if } \operatorname{rand}() \in\left[c_{p}, c_{g}\right), \\
x, \text { if rand }) \in\left[c_{g}, 1\right) .
\end{array}\right.
$$

In this equation, $\mathrm{i}=1,2, \ldots, \mathrm{M}$, where $\mathrm{M}$ is the swarm population. $\mathrm{X}_{\mathrm{i}}=\left(x_{i 1}, x_{i 2}, \ldots, x_{i d}\right)$, where $x_{i d}$ is the position value of the $\mathrm{i}$-th particle with respect to the D-th dimension of the feature space. $\mathrm{Cw}, \mathrm{Cp}$ and $\mathrm{Cg}$ are three predetermined positive constants with $\mathrm{Cw}<\mathrm{Cp}<\mathrm{Cg} . \mathrm{P}_{\mathrm{i}}=$ $\left(\mathrm{p}_{\mathrm{i} 1}, \mathrm{p}_{\mathrm{i} 2}, \ldots, \mathrm{p}_{\mathrm{id}}\right)$ denotes the best solution achieved so far by itself (pbest), and the best solution achieved so far by the whole swarm (gbest) is represented by $G_{i}=\left(g_{i 1}, g_{i 2}, \ldots\right.$, $\mathrm{g}_{\mathrm{id}}$ ). The $\mathrm{x}$ represents the particle location value in each dimention which will be retained or updated by its pbest value or the best value in each generation from random function and(), where the random number lies in between 0 and 1.

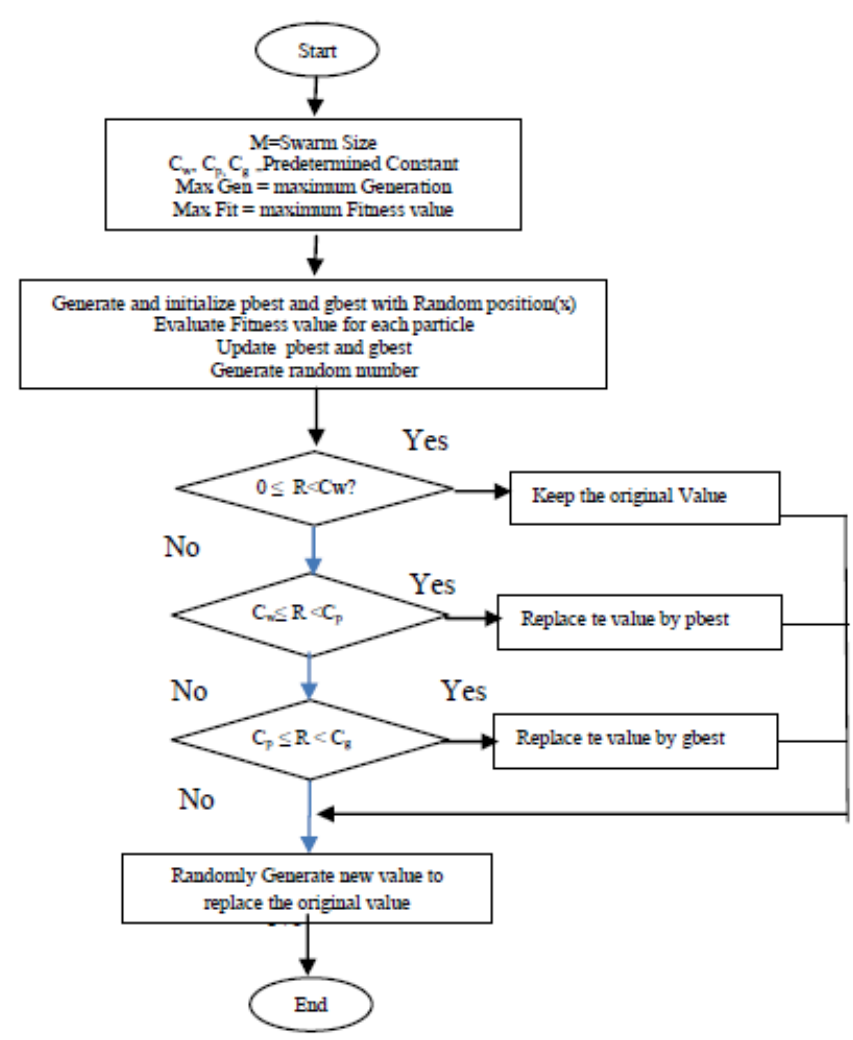

Figure-1 Flow Chart for SSO Algorithm

The proposed algorithm aims at maximizing the number of satisfied users under bandwidth constrains and ensures the quality of service.

Published By:

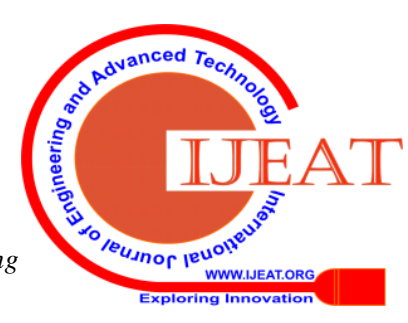


Since cognitive system contributes a dynamic spectrum allocation environment, it's essential to conduct dynamic spectrum allocation. Nevertheless, PSO suffers from premature convergence which results in the particle having difficulty in achieving the best fitness value.

In genetic algorithm, the population converged too early for an optimisation problem. In order to improve the performance and overcome the drawback of GA and PSO this paper proposes a new Simplified Swarm Optimization (SSO) algorithm whereby incorporating it with the new local search strategy which finds a better solution.

\section{IMPLEMENTATION}

Network Simulator OPNET is used to simulate $1000 \mathrm{nu}$ mber of nodes in an area of 5000 square meters which is randomly placed. Network node are categories to configure and to characterize different types of nodes similar to Servers, Gateway, Routers, Laptops, Mobile devices and IoT based Wireless Sensors. Depending on node configuration, all common protocol types such as IEEE $802.11 \mathrm{~b} / \mathrm{g} / \mathrm{n}$ are used in mixed mode in order to reflect a standard real world interaction, flexibility and network traffic are randomized. Simulation is performed with the simulation tool OPNET for 1-hour real world time and the parameters Throughput, Latency, End-To-End Delay, Average Power Consumption, Average Adaptation Time and Average Total Utility were logged in every 6 minutes for 60 minutes duration. Visual Studio IDE is used to create the user interface and compare the performance of GA, PSO and SSO. A computer with Intel ${ }^{\circledR}$ Core ${ }^{\mathrm{TM}}$ i5 processor, 4GB RAM is used to run the experiment. Simulation is performed for one hour simulation time with 10 minutes time stamps. OPNET simulation setup is given in Figure-2.

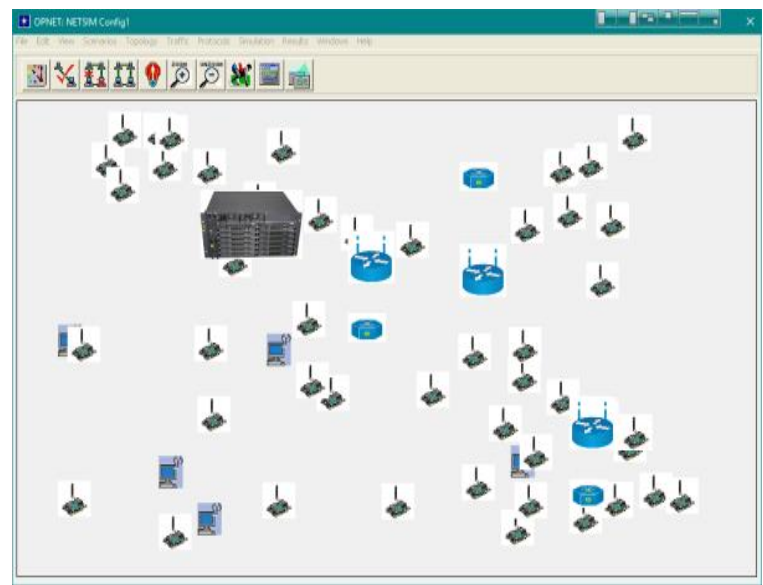

Figure-2 OPNET Simulation Setup

\section{RESULTS AND DISCUSSION}

Network metrics such as Throughput, Latency, End-ToEnd Delay, Average Power Consumption, Average Adaptation Time and Average Total Utility are measured 10 times in a simulation hour with 10 minutes timestamps. Results of existing methods GA, PSO and proposed SSO are logged in a report files and graphs are plotted based on the results for the above network metrics which is already defined. Throughput refers how much data can be transferred from sender to receiver in a given amount of time.

Throughput $=$ Number of Packets sent $/$ Time Taken. It is the actual amount of data that is successfully sent/receive in a acommunication link. It is one of the important metrics of network that dissembles the quality of the network. Measured throughput for GA, PSO and SSO are given in Table 1.

Table 1. Throughput (bps)

\begin{tabular}{|c|r|c|c|}
\hline \multirow{2}{*}{ Time Stamp } & \multicolumn{3}{|c|}{ Throughput } \\
\cline { 2 - 4 } & GA & PSO & SSO \\
\hline 1 & 2432964 & 2567453 & 2723976 (bps) \\
\hline 2 & 2403172 & 2544284 & $2704200 \quad$ (bps) \\
\hline 3 & 2373415 & 2521174 & 2684539 (bps) \\
\hline 4 & 2343530 & 2497877 & 2664740 (bps) \\
\hline 5 & 2313857 & 2474739 & 2645007 (bps) \\
\hline 6 & 2284000 & 2451635 & 2625395 (bps) \\
\hline 7 & 2254328 & 2428708 & 2605601 (bps) \\
\hline 8 & 2224380 & 2405396 & 2586034 (bps) \\
\hline 9 & 2194544 & 2382467 & 2566082 (bps) \\
\hline 10 & 2165184 & 2358778 & 2546276 (bps) \\
\hline
\end{tabular}

The parameter Throughput is higher for SSO when compared with GA and PSO as per the observation of the graph in Figure-2 as well as in Table 1 observations. SSO takes an average time (or) Throughtput of 2723976 (bps) which is higher than other Throughtput 2567453 (bps) for PSO and Throughtput 2432964 (bps) and 80 of GA during first Time Stamp. This higher value reflects the higher efficiency of the proposed method SSO.

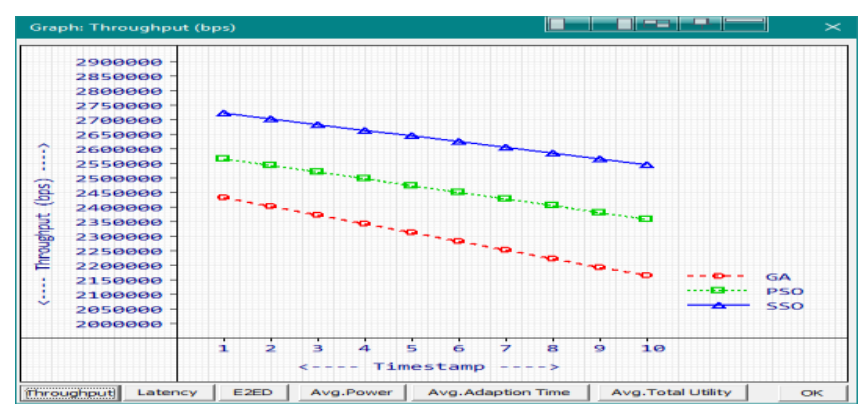

Figure-2. Throughput (bps)

\section{Latency:}

Latency $=$ Propagation Delay + Serialization Delay Propagation Delay $=$ Distance $/$ Speed Serialization Delay $=$ Packet size (bits) / Transmission rate(bps)

Latency is the time it takens for a packet to get across the communication link form sender to receiver. It is measured in units of time-ms (Milli Seconds)

Measured latency for GA, PSO and SSO are given in table 2 .

Table. 2. Latency (mS)

\begin{tabular}{|c|l|l|c|}
\hline \multirow{2}{*}{ Time Stamp } & \multicolumn{3}{|c|}{ Latency } \\
\cline { 2 - 4 } & GA & PSO & SSO \\
\hline 1 & 60 & 50 & $31(\mathrm{mS})$ \\
\hline 2 & 55 & 51 & $40(\mathrm{mS})$ \\
\hline 3 & 58 & 51 & $32(\mathrm{mS})$ \\
\hline 4 & 62 & 51 & $32(\mathrm{mS})$ \\
\hline 5 & 61 & 43 & $32(\mathrm{mS})$ \\
\hline 6 & 54 & 42 & $39(\mathrm{mS})$ \\
\hline
\end{tabular}




\begin{tabular}{|l|l|l|l|}
\hline 7 & 63 & 49 & $36(\mathrm{mS})$ \\
\hline 8 & 62 & 44 & $32(\mathrm{mS})$ \\
\hline 9 & 59 & 50 & $40(\mathrm{mS})$ \\
\hline 10 & 62 & 49 & $32(\mathrm{mS})$ \\
\hline
\end{tabular}

The latency value is lesser for SSO when compared with the GA and PSO according to the observation of the table 2. In the first time stamp, SSO takes an average period of 31(mS) which is less than other timings 50 (mS) for PSO and $60(\mathrm{mS})$ for GA. The lower value indicates the proposed SSO is higher in efficiency.

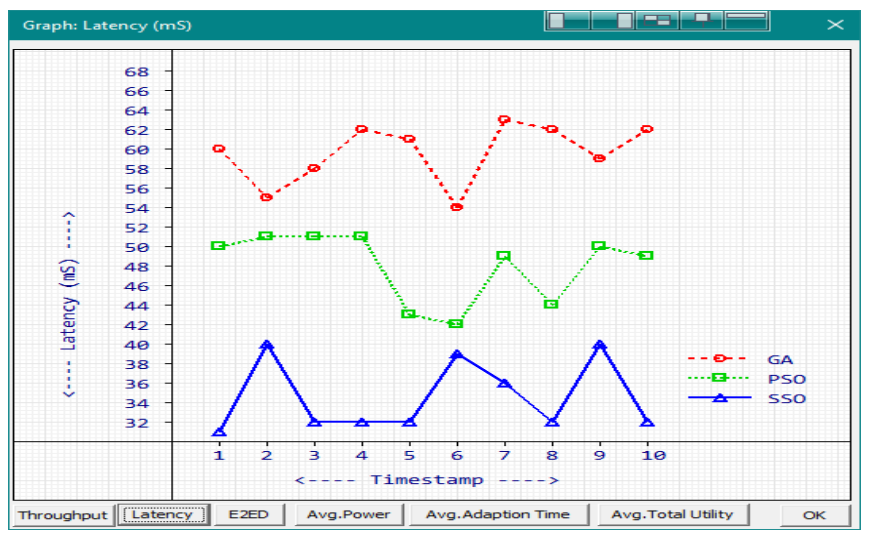

Figure-3. Latency (mS)

\section{End-to-End Delay:}

The formula for end-to-end Delay $\mathrm{I}=(\mathrm{P}+(\mathrm{N}-1))^{*} \mathrm{~L} / \mathrm{R}$ where, $\mathrm{P}=$ Packets, $\mathrm{N}=$ Number of links, $\mathrm{L}=$ Packet Length, $\mathrm{R}=$ Transmission Rate.

End-to-End delay is the time needed for the destination application to get the packet generated by the source application. End-to-end delay consists of two parts: endpoint application delay and network delay. End-point delay is the delay introduced by the end-point applications. Network delay is defined as the time the first bit of the packet is put on the wire at the source reference point to the time the last bit of the packet is received at the receiver reference point.

Table-3. End-to-End Delay (mS)

\begin{tabular}{|c|c|c|c|}
\hline \multirow{2}{*}{ Time Stamp } & \multicolumn{3}{|c|}{ End-to-End Delay } \\
\cline { 2 - 4 } & GA & PSO & SSO \\
\hline 1 & 305 & 277 & $260(\mathrm{mS})$ \\
\hline 2 & 333 & 303 & $284(\mathrm{mS})$ \\
\hline 3 & 350 & 324 & $314(\mathrm{mS})$ \\
\hline 4 & 378 & 348 & $335(\mathrm{mS})$ \\
\hline 5 & 398 & 376 & $361(\mathrm{mS})$ \\
\hline 6 & 429 & 387 & $378(\mathrm{mS})$ \\
\hline 7 & 453 & 416 & $410(\mathrm{mS})$ \\
\hline 8 & 482 & 445 & $441(\mathrm{mS})$ \\
\hline 9 & 512 & 476 & $467(\mathrm{mS})$ \\
\hline 10 & 527 & 491 & $482(\mathrm{mS})$ \\
\hline
\end{tabular}

In contrast with the GA and PSO, the End - to - End Delay parameter I s lower for SSO as per the observation from the Table-3 and the graph in Figure-4. During the period of first time stamp, SSO takes an average time of 260 (mS) which is lower than other timings PSO 277 (mS) and $3059 \mathrm{mS}$ ) for GA. This lower value reflects the proposed SSO has higher efficiency.

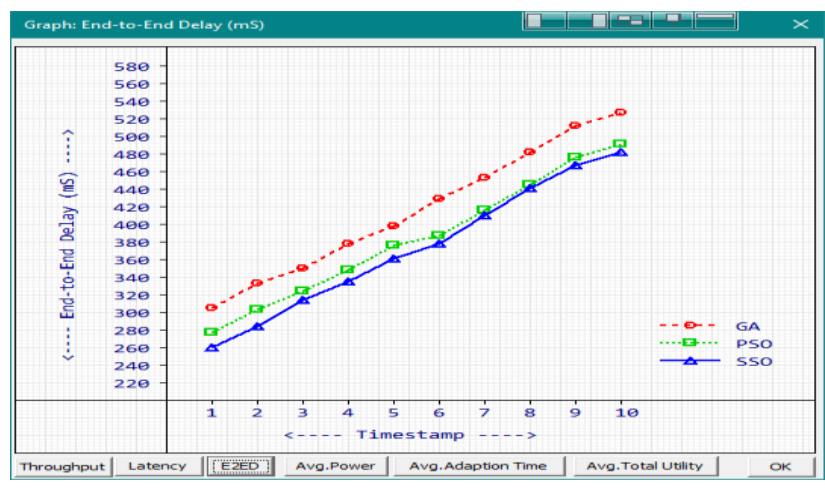

Figure-4. End-to-End Delay (mS)

\section{Average Power Consumption:}

Power consumption is measured in mWh. The power $P=V \times I$, where $\mathrm{V}$ refers the Voltage and I refers the current. OPNET is capable of measuring the average power utilized in a node by tracking the power consumption of all nodes. OPNET provides the initial power randomly to replicate the real-world environment. OPNET handles the voltage and current information of all nodes as a set $P=\left\{\left(V_{1}, I_{1}\right),\left(V_{2}, I_{2}\right) \ldots\left(V_{n}, I_{n}\right)\right\}$. This set is maintained throughout the simulation process. OPNET supports heterogeneous network environments, thus each node can have different V,I parameters. Initially the simulator allots V and I parameters randomly for every node. The average power consumption for a network transaction is calculated using the following formula.

$$
P_{a}=\frac{1}{n} \sum_{\mathrm{i}=1}^{n}\left(V_{\mathrm{i}} \times I_{\mathrm{i}}\right)
$$

where $n$ is the number of nodes in the simulation. Measured Average Power Consumption for GA, PSO and SSO are given in Table-4.

Table-4. Average Power Consumption (mW)

\begin{tabular}{|c|c|c|c|}
\hline \multirow{2}{*}{ Time Stamp } & \multicolumn{4}{|c|}{ Average Power Consumption } \\
\cline { 2 - 4 } & GA & PSO & SSO \\
\hline 1 & 80 & 69 & $54(\mathrm{~mW})$ \\
\hline 2 & 91 & 77 & $61(\mathrm{~mW})$ \\
\hline 3 & 104 & 88 & $71(\mathrm{~mW})$ \\
\hline 4 & 115 & 96 & $79(\mathrm{~mW})$ \\
\hline 5 & 129 & 105 & $90(\mathrm{~mW})$ \\
\hline 6 & 136 & 110 & $91(\mathrm{~mW})$ \\
\hline 7 & 156 & 127 & $97(\mathrm{~mW})$ \\
\hline 8 & 166 & 129 & $109(\mathrm{~mW})$ \\
\hline 9 & 176 & 138 & $118(\mathrm{~mW})$ \\
\hline 10 & 192 & 141 & $131(\mathrm{~mW})$ \\
\hline
\end{tabular}

The main difference with the GA and PSO, the parameter Average Power Consumption $(\mathrm{mW})$ is lower for SSO as per the observation from Table-4 and Ffigure-5. The duration taken for Average Power Consumption the first time stamp in Table-4 reflects that SSO takes an average time of $54(\mathrm{~mW})$ which is lesser than other timings such as $69(\mathrm{~mW})$ for PSO and $80(\mathrm{~mW})$ for GA. This lesser value shows the higher efficiency of the proposed method SSO. 


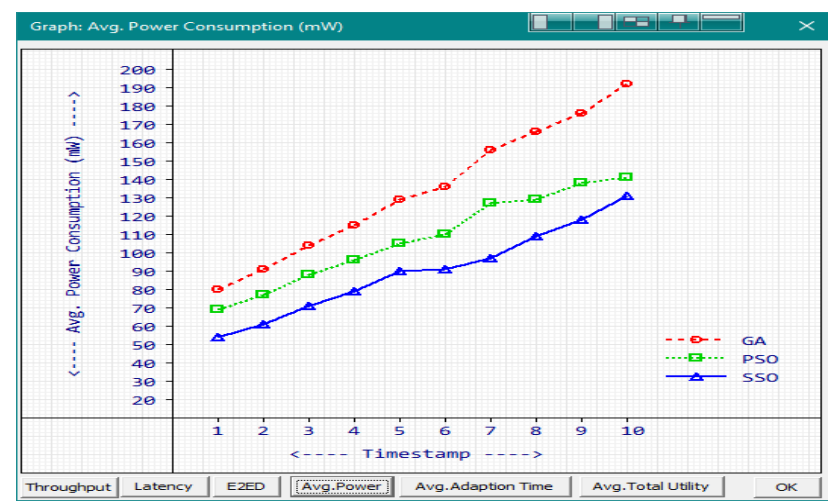

Figure-5. Average Power Consumption (mW)

\section{Average Adaptation Time:}

Adaptation time refers the time duration taken for a network cluster with a base frequency to append a new node. Let $\alpha$ be a set of adaptation times of new nodes $\left\{\alpha_{N_{1}}, \alpha_{N_{2},-} \alpha_{N_{\mathrm{m}}}\right\}$, the average adaptation time is calculated as $T_{\alpha}=\frac{\sum_{1=1}^{\mathrm{m}} \alpha_{N_{i}}}{n}$ added the network band. Measured Average Adaptation Time for GA, PSO and SSO are given in Table-5.

Table-5. Average Adaptation Time (mS)

\begin{tabular}{|c|c|c|c|}
\hline \multirow{2}{*}{$\begin{array}{l}\text { Time } \\
\text { Stamp }\end{array}$} & \multicolumn{3}{|c|}{ Average Adaptation Time } \\
\cline { 2 - 4 } & GA & PSO & SSO \\
\hline 50 & 120 & 107 & $98(\mathrm{mS})$ \\
\hline 100 & 601 & 553 & $535(\mathrm{mS})$ \\
\hline 150 & 1098 & 1002 & $958(\mathrm{mS})$ \\
\hline 200 & 1584 & 1448 & $1393(\mathrm{mS})$ \\
\hline 250 & 2069 & 1867 & $1825(\mathrm{mS})$ \\
\hline 300 & 2551 & 2307 & $2265(\mathrm{mS})$ \\
\hline 350 & 3022 & 2784 & $2708(\mathrm{mS})$ \\
\hline 400 & 3534 & 3218 & $3122(\mathrm{mS})$ \\
\hline 450 & 3988 & 3656 & $3562(\mathrm{mS})$ \\
\hline 500 & 4538 & 4092 & $3974(\mathrm{mS})$ \\
\hline
\end{tabular}

Unlike the GA and PSO, the Average Adaptation Time $(\mathrm{mS})$ parameter is lesser for SSO as per the observation from the Figure- 6 and the Table-5. SSO takes an Average Adaptation Time of 98 (mS) which during the first time stamp is lesser than other timings107 (mS) for PSO and 120 $(\mathrm{mS})$ for GA. This lesser value reflects the higher efficiency of the proposed SSOmethed.

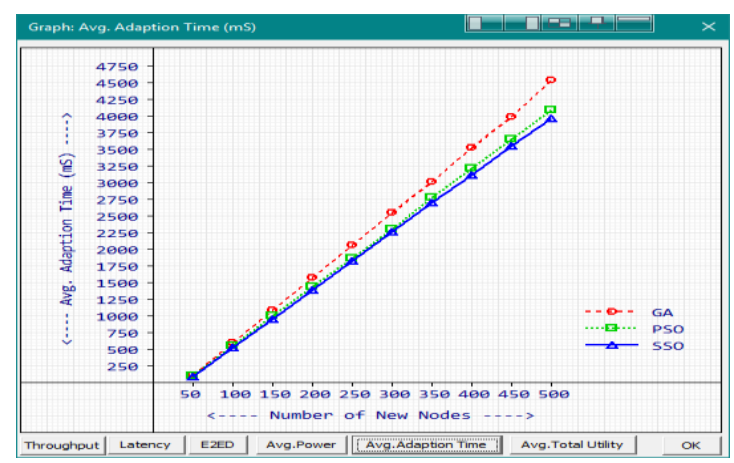

Figure-6. Average Adaptation Time (mS)

\section{Average Total Utility:}

Average Total Utility is calculated by averaging the total utility of the network resource (spectrum) throughout the simulation. Total utility is calculated for every time slices between two timestamps and the average of these values is represented as average total utility. Measured Average Adaptation Time for GA, PSO and SSO are given in Table-6.

Table-6. Average Total Utility (\%)

\begin{tabular}{|c|l|c|c|}
\hline \multirow{2}{*}{ Time Stamp } & \multicolumn{3}{|c|}{ Average Total Utility } \\
\cline { 2 - 3 } & GA & PSO & SSO \\
\hline 50 & 82.01 & 85.04 & $87.70(\%)$ \\
\hline 100 & 81.23 & 84.25 & $86.86(\%)$ \\
\hline 150 & 80.32 & 83.19 & $85.74(\%)$ \\
\hline 200 & 79.23 & 82.40 & $85.11(\%)$ \\
\hline 250 & 79.01 & 81.26 & $84.07(\%)$ \\
\hline 300 & 77.25 & 80.13 & $83.03(\%)$ \\
\hline 350 & 76.27 & 79.68 & $82.56(\%)$ \\
\hline 400 & 76.36 & 78.83 & $81.55(\%)$ \\
\hline 450 & 74.07 & 77.36 & $79.91(\%)$ \\
\hline 500 & 73.77 & 76.51 & $79.09(\%)$ \\
\hline
\end{tabular}

The observation from the Figure-7 and Table-6 the Average Total Utility percentage is higher for SSO when compared with the GA and PSO. SSO takes an Average Total Utilityof 87.70 (\%) which is higher than other timings 85.04 (\%) for PSO and 82.01(\%) for GA during first time stamp. This higher value reflects the higher efficiency of the proposed method SSO.

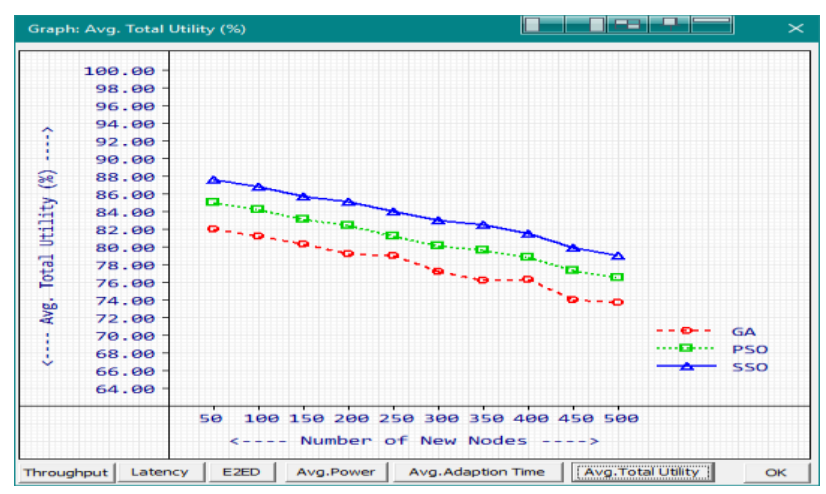

Fig. 7. Average Total Utility (\%)

\section{CONCLUSION}

As the Particle Swarm Optimization algorithm suffers from premature convergence which makes it difficult for the particle to obtain the best fitness value. In Genetic Algorithm the population convergence is too early for an optimisation issue. The theoretical and simulation results expose that the proposed Simplified Swarm Optimization Algorithm, where the new local search techniques is merged to provide a better optimization and justifies that SSO is better than GA and PSO. This model is appropriate for numerous measurements of parameters such as Throughput, Latency, End-To-End Delay, Average Power Consumption, Average Adaptation Time and Average Total Utility. For specific given period of time and optimal channel conditions, our model assumes a finite number of nodes. A new method based on Simplified Swarm Optimization technique is implemented and its performance enhancement is tested on the basis of the simulation results to enable better resource utilization.

Published By:

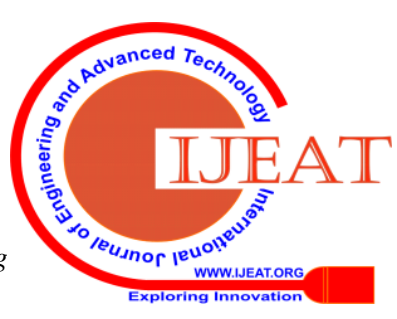


For heterogeneous environments, SSO is suggested by providing a lucide bridge for optimality between two different types of algorithms.

\section{REFERENCE}

1. Bestoun S. Ahmed, Mouayad A. Sahib, Moayad Y. Potrus, "Generating combinatorial test cases using Simplified Swarm Optimization (SSO) algorithm for automated GUI functional testing", Engineering Science and Technology, an International Journal, Volume 17, Issue 4, December 2014, Pages 218-226.

2. Changseok Bae, Wei-Chang Yeh, Noorhaniza Wahid, Yuk Ying Chung and Yao Liu, "A New Simplified Swarm Optimization (SSO) Using Exchange Local Search Scheme”, International Journal of Innovative Computing, Information and Control , Volume 8, Number 6, June 2012.

3. Changseok Bae, Wei-Chang Yeh, Noorhaniza Wahid, Yuk Ying Chung and Yao Liu, "A New Simplified Swarm Optimization (SSO) Using Exchange Local Search Scheme", International Journal of Innovative Computing, Information and Control , Volume 8, Number 6, June 2012.

4. F. Hu et al, "Full Spectrum Sharing in Cognitive Radio Networks Toward 5G: A Survey", IEEE Access PP(99):1-1 · February 2018, DOI: 10.1109 / ACCESS. 2018.2802450.

5. Farokh Koroupi, HojjatSalehinejad, SiamakTalebi, "Spectrum Assignment in Cognitive Radio Networks Using Fuzzy Logic Empowered Ants", Iranian Journal of Fuzzy Systems, Volume 10, Issue 6, November and December 2013, Pages 1-19

6. Guangyi Liu and Dajie Jiang, "5G: Vision and Requirements for Mobile Communication System towards Year 2020", Chinese Journal of Engineering Volume 2016, Article ID 5974586, 8 pages. http://dx.doi.org/10.1155/2016/5974586

7. LiseSafatly,Mario Bkassiny, Mohammed Al-Husseini, and Ali ElHajj, "Cognitive Radio Transceivers: RF, Spectrum Sensing, and Learning Algorithms Review", International Journal of Antennas and Propagation, Volume 2014, Article ID 548473, 21 pages http://dx.doi.org/10.1155/2014/548473

8. Mohammad Masoud et al, "Sensors of Smart Devices in the Internet of Everything (IoE) Era: Big Opportunities and Massive Doubts", Journal of Sensors, Volume 2019, Article ID 6514520, 26 pages, https://doi.org/10.1155/2019/6514520.

9. Nan Zhao, F. Richard Yu,Hongjian Sun, Ming Li, “Adaptive Power Allocation Schemes for Spectrum Sharing in Interference-AlignmentBased Cognitive Radio Networks", IEEE Transactions on Vehicular Technology, Volume: 65 , Issue: 5 , May 2016.

10. P. Vijayakumar and S. Malarvihi, "Green Spectrum Sharing: Genetic Algorithm Based SDR Implementation", Wireless Personal Communications volume 94, pages2303-2324(2017)

11. Raúl Chávez-Santiago et al,"5G: The Convergence of Wireless Communications",Wireless Personal Communications volume 83, pages1617-1642 (2015)

12. Richard N, Clarke, "Expanding mobile wireless capacity: The challenges presented by technology and economics, Telecommunications Policy", Volume 38, Issues 8-9, September 2014, Pages 693-708

13. Subhashree Mishra etal, "Spectrum Allocation in Cognitive Radio: A PSO-based Approach", PeriodicaPolytechnica Electrical Engineering and Computer Science, 63(1), pp. 23-29, 2019.

14. Tuan Le et al, "Survey of Promising Technologies for 5G Networks", Mobile Information Systems, Volume 2016, Article ID 2676589, 25 pages, http://dx.doi.org/10.1155/2016/2676589

15. YaoWang et al, "A Novel Spectrum Allocation Algorithm for Cognitive Radio Networks", Procedia Engineering, Volume 29, 2012, Pages 2776-2780.

\section{AUTHORS PROFILE}

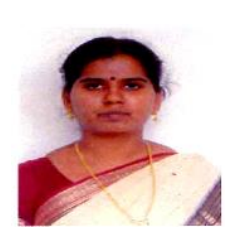

Rajalakshmi, presently a full time Research Scholar, Department of Computer Science, Bharathidasan University, Tiruchirappalli. She has completed MCA, M.Phil Degree in Computer Science and pursuing his full time Ph.D in Optimization of Spectrum Allocation in Cognitive Radio Technology. Previously, she has worked as Lecturer at Department of Computer Science, Dhanalakshmi Srinivasan College of Art \& Science for Women, Perambalur from 2002 to 2009. Then she worked as Assistant Professor at Department of Computer Science, Rover Engineering College, Elambalur from 2009 to 2013.

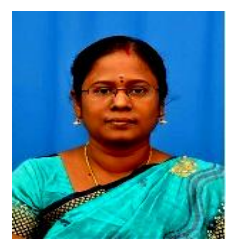

P. Sumathy, is currently Assistant Professor at Department of Computer Science, Bharathidasan University, Tiruchirappalli. She has completed M.Sc in Computer Science, M. Phil in Computer Science and Ph.D in the area of Image Retrieval. She has around 12 years of Academic Experience and 9 years of Research Experience. She specializes in Content based Image Retrieval, Multimedia Information Retrieval and Data Mining. She has published several Research papers in National and International Journals of repute. 